\title{
The stabilization of the nonlinear model arising in pulse propagation in optical fiber
}

\author{
Patricia Nunes da Silva, $\quad$ Carlos Frederico Vasconcellos ${ }^{1}$ \\ Department of Mathematical Analysis, IME, UERJ, \\ 20550-013, Rio de Janeiro, RJ, Brasil \\ E-mail: nunes@ime.uerj.br, cfredvasc@ime.uerj.br,
}

Resumo: We analyze the stabilization of the third order nonlinear Schrödinger equation in a bounded interval under the effect of a localized damping mechanism. That is, we consider the following equation:

$$
i u_{t}+\alpha u_{x x}+i \beta u_{x x x}+|u|^{2} u+i a(x) u=0 .
$$

Where $u=u(x, t)$ is a complex valued function defined in $(0, L) \times(0,+\infty)$ and $\alpha, \beta$ are real constants. This equation models a pulse propagation in a long-distance and high-speed optical fiber transmission system. Using multiplier techniques and a special uniform continuation theorem we prove the exponential decay of the total energy associated with the above system.

Key Words: exponential decay, stabilization, nonlinear Schrödinger equation

\section{Introduction}

We consider the third order nonlinear damped Schrödinger (NS) system in a bounded interval

$$
\mid \begin{aligned}
& i u_{t}+\alpha u_{x x}+i \beta u_{x x x}+|u|^{2} u+i a(x) u=0 \quad \text { in } \quad(0, L) \times(0,+\infty) \\
& u(0, t)=u(L, t)=0 \quad \text { for all } \quad t \geq 0 \\
& u_{x}(L, t)=0 \quad \text { for all } t \geq 0 \\
& u(x, 0)=u_{0} \quad \text { in } \quad(0, L) .
\end{aligned}
$$

Where $u$ is a complex valued function, $u_{0}$ belongs to $L^{2}(0, L)$, the constant $\alpha$ is a real non null constant and $\beta$ is a positive constant.

Here $a=a(x)$ is a non-negative real valued function belonging to $L^{\infty}(0, L)$ and moreover we will assume that $a(x) \geq a_{0}>0$ a.e. in an open, non-empty subset $\omega$ of $(0, L)$, where the damping is acting effectively.

The above third order nonlinear Schrödinger equation was proposed by Kodama [3] to model a pulse propagation in a long-distance and high-speed optical fiber transmission system. Kodama [3] considered the following (perturbed) nonlinear Schrödinger equation with higher-order terms (as the perturbation terms)

$$
i u_{t}+\frac{1}{2} u_{x x}+|u|^{2} u=i \epsilon\left(\beta_{1} u_{x x x}+\beta_{2}|u|^{2} u_{x}+\beta_{3} u^{2} \bar{u}_{x}\right)-i \Gamma u,
$$

where $\epsilon$ is a small real parameter $(|\epsilon| \ll 1)$ and $\beta_{1}, \beta_{2}, \beta_{3}$ and $\Gamma$ are real constants.

In [2], Chu used equation (1.2) with $\beta_{2}=\beta_{3}=\Gamma=0$ as a model for the soliton propagation in an optical fiber. He showed that the third order term can be used to reduce the mutual interaction between solitons when the fiber is operated nonlinearly.

\footnotetext{
${ }^{1}$ Visiting Professor supported by FAPERJ
} 
The present paper is devoted to study the rate of the decay of the energy associated to the system (1.1), as the time $t$ tends to $+\infty$

We consider the inner product in $L^{2}(0, L)$ defined by:

$$
(f \mid g)=\operatorname{Re} \int_{0}^{L} f(x) \overline{g(x)} d x
$$

and the inner product in $H_{0}^{1}(0, L)$ by:

$$
(f \mid g)_{1}=\operatorname{Re} \int_{0}^{L} f_{x}(x) \overline{g_{x}(x)} d x
$$

The energy is defined by:

$$
E(t)=\frac{1}{2} \int_{0}^{L}|u(x, t)|^{2} d x=\frac{1}{2}\|u(t)\|^{2} .
$$

Using the boundary conditions in (1.1) we prove that

$$
\frac{d E}{d t}=-\frac{\beta}{2}\left|u_{x}(0, t)\right|^{2}-\int_{0}^{L} a(x)|u(x, t)|^{2} d x \quad \forall t>0 .
$$

Since $\beta>0$ and by assumption on damping $a$, we observe that according to the above energy dissipation law, the energy $E(t)$ is a nonincreasing function of the time.

In section 2, for the sake of the completeness, we make a brief analysis of the linear case, that is, the system (1.1) without the term $|u|^{2} u$. We use the work of Silva-Vasconcellos [10] and some multiplier techniques when we consider the additional damping term.

In section 3, we study the system (1.1). At first, we prove the existence and uniqueness of solutions and then we answer the following questions:

Does the energy $E(t) \rightarrow 0$ as $t \rightarrow+\infty$ ? Is it possible to find a rate of decay of the energy?

We show that the energy associated to problem (1.1) decays exponentially and this decay is uniform.

Dispersive problems have been object of intensive research (see, for instance, the classical paper of Benjamin-Bona-Mahoni [1], Menzala et al. [5], Rosier [7], and references therein). Global stabilization of the generalized KdV system has been obtained by Rosier-Zhang [8]. LinaresPazoto [4] studied the stabilization of the generalized KdV system with critical exponents.

Vasconcellos-Silva [12] studied the existence, regularity of the solutions and stabilization for the Kawahara system.

\section{The Linear System}

In this section we analyze the linear system associated with system (1.1).

Taking into account the work of Silva-Vasconcellos[10], we begin by analysing existence, uniqueness, regularity of solutions and exponential decay of the energy associated to the following system:

$$
\mid \begin{aligned}
& i u_{t}+\alpha u_{x x}+i \beta u_{x x x}+i a(x) u=0 \quad \text { in } \quad(0, L) \times(0,+\infty) \\
& u(0, t)=u(L, t)=0 \quad \text { for all } t \geq 0 \\
& u_{x}(L, t)=0 \quad \text { for all } t \geq 0 \\
& u(x, 0)=u_{0} \quad \text { in } \quad(0, L) .
\end{aligned}
$$

Here $u_{0}$ belongs to $L^{2}(0, L), \alpha$ is a real non null constant and $\beta$ is a positive constant, moreover $a=a(x)$ is a non-negative real valued function belonging to $L^{\infty}(0, L)$ and $a(x) \geq a_{0}>0$ a.e. in an open, non-empty subset $\omega$ of $(0, L)$. 
Theorem 2.1. (Existence, uniqueness and regularity)

The problem (2.1) has a unique solution u belonging to $C\left([0,+\infty) ; L^{2}(0, L)\right) \cap L^{2}\left(0,+\infty ; H_{0}^{1}(0, L)\right)$. Moreover,

i) $\|u\|_{C\left([0,+\infty) ; L^{2}(0, L)\right)} \leq C\left\|u_{0}\right\|$

ii) $u_{x}(0,$.$) belongs to L^{2}(0,+\infty)$ and $\left\|u_{x}(0, .)\right\|_{L^{2}(0,+\infty)} \leq C\left\|u_{0}\right\|$

iii) For each $T>0$, $u$ belongs to $L^{2}\left(0, T ; H_{0}^{1}(0, L)\right)$ and there exists $C_{1}(L, T, \beta)>0$ such that:

$$
\|u\|_{L^{2}\left(0, T ; H_{0}^{1}(0, L)\right)} \leq C_{1}\left\|u_{0}\right\| .
$$

and the energy dissipation law,

$$
\frac{d E}{d t}=-\frac{\beta}{2}\left|u_{x}(0, t)\right|^{2}-\int_{0}^{L} a(x)|u(x, t)|^{2} d x \leq 0, \quad \forall t>0,
$$

holds.

We use semigroups theory to prove the existence and uniqueness and to show regularity of solutions we consider the multipliers techniques.

The items i), ii), iii) and the dissipation law follow as in Silva-Vasconcellos[10], considering there the parameter $\gamma=0$.

In what concerns stabilization, the following result holds.

\section{Theorem 2.2. (Stabilization for the linear case)}

Assume that $a \geq a_{0}>0$ a.e. in an open non-empty subset $\omega$ of $(0, L)$. Then, for any $L>0$, there exist $c>0$ and $\mu>0$ such that

$$
E(t) \leq c\left\|u_{0}\right\|^{2} e^{-\mu t}
$$

for all $t \geq 0$ and all solution of the system (2.1) with $u_{0} \in L^{2}(0, L)$.

To prove the above theorem we use multiplier techniques and the Holmgren's Uniqueness Theorem. This proof follows the same method developed in Menzala et al. [5] for the KdV linear system and Vasconcellos-Silva [11] for the Kawahara linear system.

\section{The System (1.1)}

In this section we analyzed our main problem.

At first and again for sake of completeness we prove the existence and uniqueness of global solution for the system (1.1)

Theorem 3.1. (Existence and uniqueness)

If $u_{0}$ belongs to $L^{2}(0, L)$, then the problem (1.1) has a unique solution $u$ belonging to $C\left([0,+\infty) ; L^{2}(0, L)\right) \cap L_{l o c}^{2}\left(0,+\infty ; H_{0}^{1}(0, L)\right)$.

We can observe, using boundary conditions, that the total energy associated to system satisfies the energy dissipation law:

$$
\frac{d E}{d t}=-\frac{\beta}{2}\left|u_{x}(0, t)\right|^{2}-\int_{0}^{L} a(x)|u(x, t)|^{2} d x \leq 0, \quad \forall t>0,
$$

So, it is sufficient to prove local (in time) existence and uniqueness for the system (1.1). Global existence will then follow from (3.1). We define a continuous mapping on a convenient Banach space and we use a fixed point theorem.

The aim of this work is to show the locally uniform exponential decay of the energy associated to the problem (1.1). We employ the method developed for the linear case, see the Theorem 2.2, however, we may not apply Holmgren's principle since we have now a semi-linear problem. So, we must consider an important result named Unique Continuation Principle (UCP) for the system (1.1), which we state below. 


\section{Theorem 3.2. (Unique Continuation Principle (UCP))}

Let $\omega$ be an open, non-empty subset of $(0, L)$.

If $u \in L^{2}\left(0, T ; H^{3}(0, L)\right) \cap L^{2}\left(0, T ; H_{0}^{1}(0, L)\right) \cap L^{\infty}\left(0, T ; L^{2}(0, L)\right)$ solves

$$
\mid \begin{aligned}
& i u_{t}+\alpha u_{x x}+i \beta u_{x x x}+\lambda|u|^{2} u=0 \quad \text { in } \quad(0, L) \times(0,+\infty) \\
& u(0, t)=u(L, t)=0 \quad \text { for all } t \geq 0 \\
& u_{x}(L, t)=0 \quad \text { for all } t \geq 0 \\
& u \equiv 0 \quad \text { in } \quad \omega \times(0, T)
\end{aligned}
$$

with $\lambda>0$ and $T>0$, then necessarily $u \equiv 0$ in $(0, L) \times(0, T)$.

The proof of the above theorem follows from the result due to Saut-Scheurer [9].

Now, we can enunciate our main theorem:

\section{Theorem 3.3. The stabilization for system (1.1)}

Assume the open subset $\omega$ of $(0, L)$ is such that the $(U C P)$ above holds. Let $a=a(x)$ be a non-negative function belonging to $L^{\infty}(0, L)$ such that $a(x) \geq a_{0}>0$ a.e. in $\omega$. Then, for all $R>0$, there exist $c(R)=c>0$ and $\mu(R)=\mu>0$ such that

$$
E(t) \leq c\left\|u_{0}\right\|^{2} e^{-\mu t}
$$

for all $t \geq 0$ and any solution of (1.1) with $u_{0} \in L^{2}(0, L)$ and $\left\|u_{0}\right\| \leq R$.

To prove the theorem 3.3 we use (UCP) and therefore we need to obtain a gain of regularity for the solutions of the system (3.2), for this, we use a similar method to that found in Pazoto [6].

\section{References}

[1] T.B.Benjamin, J.L.Bona and J.J.Mahony, Model equations for long waves in nonlinear dispersive systems, Philos. Trans. Roy. Soc. London, Ser. A 272, 1972, 47-78.

[2] P. L. Chu, Effect of third-order dispersion of optical fibre on soliton interaction, Electron. Lett. 21, 1985, 228-229.

[3] Y. Kodama, Optical solitons in a monomode fiber, J. Statist. Phys. 39, 1985, 597-614.

[4] F.Linares and A.F.Pazoto, On the exponential decay of the critical generalized Korteweg-de Vries with localized damping, Proc. Amer. Math. Soc., 135, 5, 2007, 1515-1522.

[5] G.P.Menzala, C.F.Vasconcellos and E.Zuazua, Stabilization of the Korteweg-de Vries equation with localized damping, Quarterly Applied Math., vol. LX, 1, 2002, 111-129.

[6] A.F.Pazoto, Unique Continuation and Decay for the Korteweg-De Vries Equation with Localized Damping, ESAIM Control Optim. Calc. Var., 11, 2005, 473-486.

[7] L.Rosier, Exact boundary controllability for the Korteweg-de Vries equation on a bounded domain, ESAIM Control Optim. Calc. Var., 2, 1997, 33-55.

[8] L.Rosier and B.Y.Zhang, Global Stabilization of the Generalized Korteweg-De Vries Equation Posed on a Finite Domain, SIAM Journal on Control and Optimization, 45, 3, 2006, 927-956.

[9] J.C. Saut and B. Scheurer, Unique continuation for some evolution equations, J. Diff. Equation, 66, 1987, 118-139. 
[10] P. N. da Silva and C. F. Vasconcellos, On the stabilization and controllability for a third order linear equation, Portugal.Math. 68, 2011, 279-296

[11] C. F. Vasconcellos and P. N. da Silva, Stabilization of the Linear Kawahara Equation with Localized Damping, Asymptotic Analysis, 58, 4, 2008, 229-252.

[12] C. F. Vasconcellos and P. N. da Silva, Stabilization of the Kawahara Equation with Localized Damping, ESAIM Control Optim. Calc. Var., 17, 2011, 102-116. 